\title{
Contraceptive Choices, Pregnancy Rates, and Outcomes in a Microbicide Trial
}

\author{
Sengeziwe Sibeko, MBChB, FCOG, Cheryl Baxter, MSc, Nonhlanhla Yende, MSc, \\ Quarraisha Abdool Karim, PhD, and Salim S. Abdool Karim, MBChB, PhD, on behalf of the Centre for \\ the AIDS Programme of Research in South Africa (CAPRISA) 004 Trial Group
}

OBJECTIVE: Women who become pregnant during the conduct of biomedical human immunodeficiency virus prevention trials are taken off the study product for safety reasons. High pregnancy rates can compromise statistical integrity in these trials. The comprehensive contraceptive curriculum developed for the Centre for the AIDS Programme of Research in South Africa

From the Centre for the AIDS Programme of Research in South Africa (CAPRISA), Nelson R. Mandela School of Medicine, University of KwaZuluNatal, Durban, South Africa; and the Department of Epidemiology, Mailman School of Public Health, Department of Epidemiology, Columbia University, New York, New York.

The CAPRISA 004 tenofovir gel trial was supported by the Centre for the AIDS Programme of Research in South Africa (CAPRISA), the US Agency for International Development (USAID), FHI (cooperative agreement GPO-A-OO05-00022-00, contract 132119), and the Technology Innovation Agency (formerly known as LIFElab), a biotechnology centre of the South African Department of Science and Technology. CONRAD provided support with product manufacturing and packaging and Gilead Sciences provided tenofovir used in the production of gel. The Columbia University-Southern African Fogarty AIDS International Training and Research Programme (AITRP), funded by the Fogarty International Center, National Institutes of Health (grant D43TW00231), supported the training of clinical trial staff, including Sengeziwe Sibeko.

The authors thank the women enrolled in the trial for their willingness to participate and to remain in follow up over the study duration. The support of their communities is also gratefully acknowledged. The authors also thank the members of the CAPRISA Research Support Group at the CAPRISA Vulindlela and eThekwini Clinical Research Sites; the staff at the CAPRISA eThekwini and Vulindlela Clinical Research Sites in implementing this trial; and CAPRISA Data Management and Statistics staff for management and quality assurance of case report forms.

Corresponding author: Sengeziwe Sibeko, MBChB, FCOG, CAPRISA, 2nd Floor, Doris Duke Medical Research Institute, Nelson R. Mandela School of Medicine, University of KwaZulu-Natal, Private Bag X7, Congella 4013, Durban, South Africa; e-mail: sibekos1@ukzn.ac.za.

\section{Financial Disclosure}

Dr. Q. Karim is the coprincipal investigator of the HPTN Prevention Leadership Group (National Institutes of Health and National Institute of Allergy and Infectious Diseases U01 AI068619), and Dr. S. Karim was the Protocol Chair of the HPTN 035 trial, which was supported by the National Institutes of Health (grants U01AI46749 and U01AI068633). The other authors did not report any potential conflicts of interest.

(C) 2011 by The American College of Obstetricians and Gynecologists. Published by Lippincott Williams \& Wilkins.

ISSN: 0029-7844/10
(CAPRISA) 004 trial was evaluated for its ability to enhance contraceptive uptake, reduce pregnancy rates, and preserve statistical integrity.

METHODS: Contraceptive- and pregnancy-related eligibility criteria were specified in the protocol. We enrolled women who opted for a nonbarrier method of contraceptive and provided hormonal contraceptives onsite at no cost. At each monthly study visit, we provided pregnancy prevention counseling and performed pregnancy testing. Study product was withheld on pregnancy diagnosis, but women continued with monthly follow-up.

RESULTS: Contraceptive use was high throughout the study with $100 \%$ uptake at baseline and $94.71 \%$ use after a mean of 18 months follow-up at exit. Injectable progestins, particularly medroxyprogesterone acetate, remained the preferred choice of contraceptive. After 30 months of follow-up, 54 pregnancies were reported out of 889 participants, giving a pregnancy incidence rate of 3.95 per 100 woman-years $(95 \%$ confidence interval 2.96-5.17). Of all pregnancies, two thirds $(64.81 \%)$ resulted in a full-term live birth, whereas $18.52 \%$ and $11.11 \%$ pregnancies culminated as miscarriage and terminated pregnancies, respectively. There were no congenital anomalies in the early neonatal period. Pregnancies resulted in $1.56 \%$ of woman-years of study follow-up lost as a result of temporary product withdrawal.

CONCLUSION: The CAPRISA 004 contraceptive curriculum was an effective strategy for maintaining low pregnancy rates, thereby minimizing product withdrawal and loss of follow-up time.

(Obstet Gynecol 2011;118:895-904)

DOI: 10.1097/AOG.0b013e31822be512

LEVEL OF EVIDENCE: III

In 2009, there were an estimated 33.3 million human immunodeficiency virus (HIV)-infected people globally. ${ }^{1} \mathrm{~A}$ distinctive feature of this epidemic in the 21 st century is its increasing burden on young women. In sub-Saharan Africa, women aged 15-24 years are as much as eight times more likely to be 
HIV-positive than men of the same age. ${ }^{2}$ Although condoms are an effective HIV prevention strategy, many women are unable to successfully negotiate their use with their male partners, making an HIV prevention method that women can initiate and control a priority. Since 1990, various women-initiated candidate biomedical microbicides have been tested in effectiveness trials ${ }^{3}$ to assess their effect on preventing HIV infection, and several more trials are underway or planned. ${ }^{4}$

All biomedical microbicide trials, irrespective of the product being investigated, share common challenges. Because these trials enroll mostly women within the reproductive age group, one of the greatest challenges has been the high pregnancy rates, ranging from 21 to 76 per 100 personyears. $^{5-10}$ Women who become pregnant during clinical trials of experimental drugs must be taken off the product for safety reasons. Frequent and prolonged product withdrawal can compromise statistical integrity in these trials. ${ }^{6}$

Although all efforts are made to limit exposure to experimental drugs during pregnancy, some women may use the study product during the first weeks of pregnancy. Therefore, it is important to monitor the safety of the study product on pregnancy rates and outcomes. Although little is known about the safety of tenofovir gel when used during pregnancy, the oral formulation, Tenofovir disoproxil fumarate, which has been widely used by HIV-infected individuals for treatment, is designated as a pregnancy category B drug in the United States and has a reassuring teratogenic profile. Congenital anomaly rates at 2.72 per 100 live births are comparable with those in the Centers for Disease Control and Prevention's population-based birth defects surveillance system in the United States and to the rates of other antiretroviral drugs in the Antiretroviral Pregnancy Registry. ${ }^{11}$

With these challenges in mind, we hypothesized that an intensive comprehensive contraceptive curriculum is an effective strategy for enhancing contraceptive uptake, reducing pregnancy rates, minimizing inadvertent in utero drug exposure, and thereby maintaining statistical integrity and fetal safety in biomedical HIV prevention trials. We provide an analysis of contraceptive choices, pregnancy rates, and outcomes from the Centre for the AIDS Programme of Research in South Africa (CAPRISA) 004 tenofovir gel trial.

\section{MATERIALS AND METHODS}

CAPRISA 004, a phase IIb placebo-controlled, double-blind randomized clinical trial, was conducted to assess safety and effectiveness of 1\% vaginal tenofovir gel to reduce HIV acquisition in women at one rural (Vulindlela) and one urban (eThekwini) clinic in South Africa. ${ }^{12}$ A total of 889 nonpregnant HIVuninfected women between the ages of 18 and 40 years were eligibly enrolled and followed up for an average of 18 months (range 12-30 months), 611 from the rural site and 278 from the urban site. The goal was to enroll up to 1,250 women over approximately 18 months and to continue follow-up until 92 incident infections were observed. This number of events was expected to provide $90 \%$ power to detect a $50 \%$ effect (using a two-sided $\alpha=0.05$ significance level test). Participants were accrued over 19 months from May 2007 to January 2009. Women were randomly assigned in equal proportions to one of two study arms: tenofovir gel and placebo gel within 30 days of the screening visit. To facilitate blinding, each participant was randomly assigned within one of six different groups (designated by an alphanumeric variable, eg, A, B, C, D, E, and F) in a 1:1:1:1:1:1 allocation ratio. Three groups corresponded to the placebo and three to tenofovir gel. This analysis includes all women enrolled into CAPRISA 004 and included in the intention-to-treat analysis.

The main outcome of this analysis was pregnancy incidence rates. Other outcomes included contraceptive uptake and adherence, pregnancy outcomes, and time off study product. We developed tools and aids to support the trial's comprehensive contraceptive curriculum, which were used from the screening visit until study termination. We used these tools to train clinical staff on contraceptive counseling for the trial participants and to guide clinical staff with regard to all pregnancy-related procedures including study product withdrawal and resumption.

Clinical staff conducted urine pregnancy tests at screening and pregnant women were excluded. As part of eligibility criteria, we assessed a participant's contraceptive needs and pregnancy intentions over the study duration. Only women who were not planning to become pregnant sometime over the duration of the study and agreed to use a nonbarrier method of contraception for the duration of the study were offered enrollment into the study. Other nonpregnancy or contraceptive-related exclusions are provided elsewhere. ${ }^{13}$ We repeated pregnancy tests at enrollment if more than 21 days had lapsed between the screening and enrollment visits.

We provided hormonal contraceptives onsite at no cost, including progesterone-containing injectables (depot-medroxyprogesterone acetate and norethisterone enanthate and combined oral contraceptives). 
Women were referred to the nearest family planning institution per referral guidelines issued by the South African Department of Health if they opted for a nonhormonal method such as an intrauterine device or tubal ligation. Contraceptive choice was recorded on a family planning card and an individual contraceptive history log that was updated at each study visit and was retained in the participant binder. Barrier methods including both male and female condoms were provided at each study visit but were promoted principally as an HIV risk reduction method.

Trained clinical study staff provided individual contraceptive counseling as part of routine monthly follow-up procedure. Contraceptive counseling was individualized and tailored according to each woman's needs. We used a contraceptive log to indirectly assess compliance with a method. To enhance continual supply, participants' contraceptive visits were scheduled to coincide with their regular monthly study visits whenever possible. Participants who chose to receive their contraception from a government family planning clinic were required to bring their signed Department of Health family planning cards to each study visit for study data collection on use of contraceptive methods.

Urine pregnancy testing was conducted monthly. Participants testing positive for pregnancy continued with monthly follow-up visits but study product was temporarily withheld. A woman continued to have monthly pregnancy tests for the first 3 months of pregnancy to exclude unrecognized early preclinical pregnancy loss. Blood $\beta$-human chorionic gonadotropin quantification was done to confirm ambiguous urine results. Product was resumed after a live birth or once the chemical tests (urine, blood, or both) reverted to negative after pregnancy end.

On pregnancy cessation, the clinical staff collected data on pregnancy complications and docu-

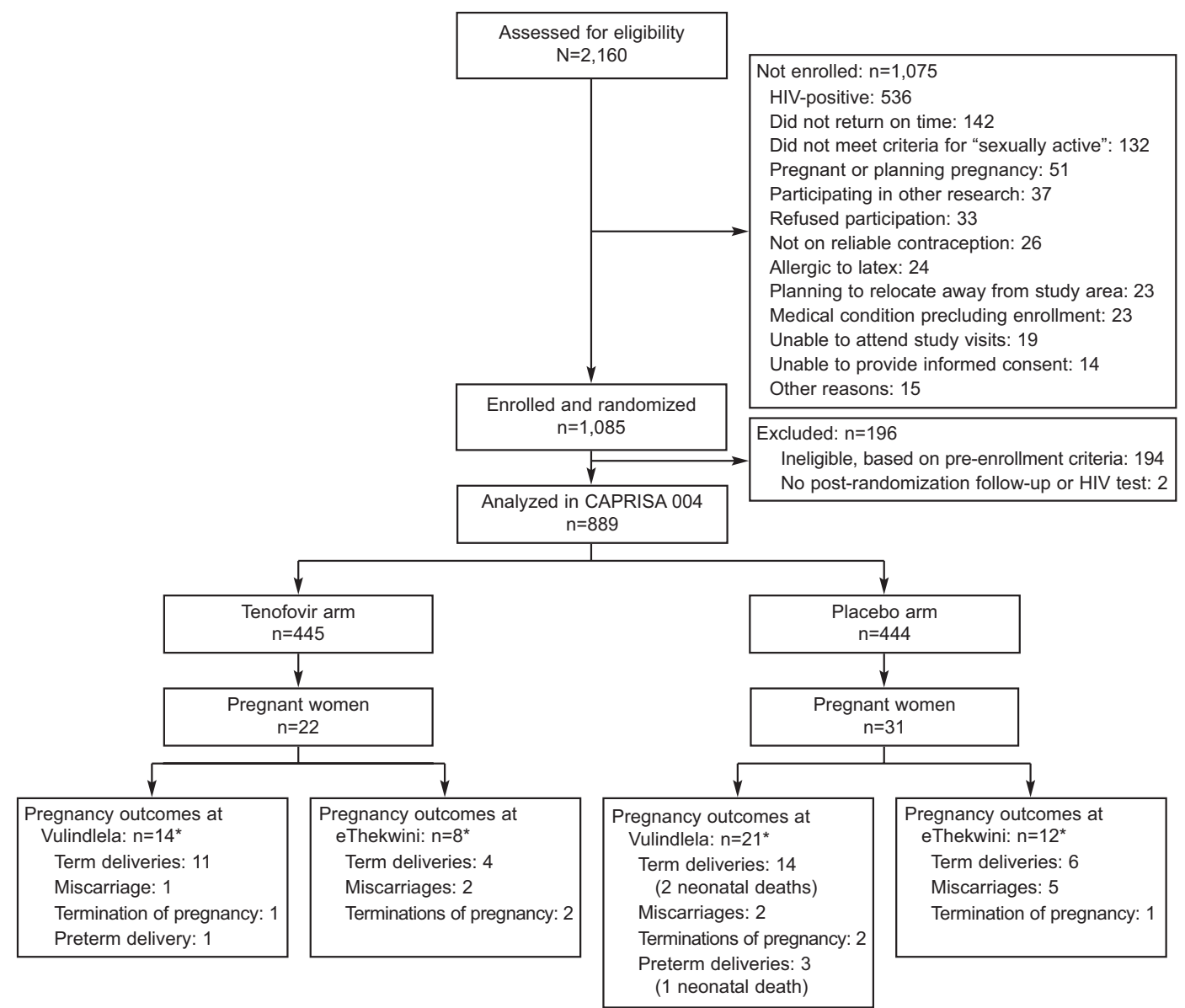

Fig. 1. Screening, enrollment, and pregnancy outcomes after 30 months of follow-up in the Centre for the AIDS Programme of Research in South Africa (CAPRISA) 004 tenofovir gel trial by study arm and site. *There were 55 pregnancy outcomes from 54 pregnancies. One woman was pregnant twice, and one gave birth to twins.

Sibeko. Contraceptive Choices and Pregnancy Rates. Obstet Gynecol 2011. 
Table 1. Baseline Demographic Characteristics and Sexual Behavior by Study Arm

\begin{tabular}{|c|c|c|c|c|}
\hline Variable & All & Tenofovir Arm & Placebo Arm & $P$ \\
\hline Site & & & & .77 \\
\hline eThekwini & 278 & $137(30.8)$ & $141(31.8)$ & \\
\hline Vulindlela & 611 & $308(69.2)$ & $303(68.2)$ & \\
\hline Age $(y)$ & $23.88 \pm 5.11$ & $24.17 \pm 5.26$ & $23.59 \pm 4.94$ & .13 \\
\hline \multicolumn{5}{|l|}{ Marital status } \\
\hline Married & 50 & $26(5.84)$ & $24(5.41)$ & .92 \\
\hline Other & 56 & $29(6.52)$ & $27(6.08)$ & \\
\hline Stable & 783 & $390(87.64)$ & $393(88.51)$ & \\
\hline \multicolumn{5}{|l|}{ Highest education } \\
\hline No schooling & 4 & $2(0.45)$ & $2(0.45)$ & .30 \\
\hline Primary schooling complete & 10 & $6(1.35)$ & $4(0.90)$ & \\
\hline Primary school not complete & 27 & $19(4.27)$ & $8(1.80)$ & \\
\hline High school complete & 301 & $150(33.71)$ & $151(34.01)$ & \\
\hline High school not complete & 483 & $238(53.48)$ & $245(55.18)$ & \\
\hline Tertiary education complete & 18 & $11(2.47)$ & $7(1.58)$ & \\
\hline Tertiary education not complete & 46 & $19(4.27)$ & $27(6.08)$ & \\
\hline \multicolumn{5}{|l|}{ Number of children } \\
\hline 0 & 197 & $90(20.23)$ & $107(24.10)$ & .42 \\
\hline 1 & 470 & $238(53.48)$ & $232(52.25)$ & \\
\hline 2 & 147 & $73(16.40)$ & $74(16.67)$ & \\
\hline 3 & 50 & $30(6.74)$ & $20(4.51)$ & \\
\hline 4 or more & 25 & $14(3.15)$ & $11(2.48)$ & \\
\hline \multicolumn{5}{|l|}{ Sex acts in past $30 \mathrm{~d}$} \\
\hline 0 & 22 & $11(2.47)$ & $11(2.48)$ & .13 \\
\hline $1-5$ & 360 & $186(41.80)$ & $174(39.19)$ & \\
\hline $6-10$ & 299 & $134(30.11)$ & $165(37.16)$ & \\
\hline 11 or more & 208 & $114(25.62)$ & $94(21.17)$ & \\
\hline \multicolumn{5}{|l|}{ Casual partner in past $30 \mathrm{~d}$} \\
\hline 0 & 826 & $412(92.58)$ & $414(93.24)$ & .96 \\
\hline 1 & 43 & $23(5.17)$ & $20(4.51)$ & \\
\hline 2 or more & 20 & $10(2.25)$ & $10(2.25)$ & \\
\hline \multicolumn{5}{|l|}{ Condom use during sex } \\
\hline Always & 259 & $128(28.76)$ & $131(29.51)$ & .81 \\
\hline Occasionally & 630 & $317(71.24)$ & $313(70.50)$ & \\
\hline \multicolumn{5}{|l|}{ Contraception } \\
\hline Injectable & 730 & 359 (80.67) & $371(83.56)$ & .29 \\
\hline Oral & 138 & $73(16.40)$ & $65(14.64)$ & \\
\hline Tubal ligation & 20 & $13(2.92)$ & $7(1.58)$ & \\
\hline Hysterectomy & 1 & $0(0.0)$ & $1(0.23)$ & \\
\hline
\end{tabular}

Data are $\mathrm{n}(\%)$ or mean \pm standard deviation unless otherwise specified.

mented pregnancy outcomes. Pregnant women presented their newborns once in the early postpartum period to the study clinician for assessment of abnormalities, if any. Case reports of each pregnancy were discussed during the Protocol Safety Review Team meetings. The Protocol Safety Review Team comprised the principal investigators, the study obstetrician, the study and site clinicians, project directors, and an independent clinician. This team met regularly, initially every 3 months then every 2 months, to review blinded safety information from the study.

The trial (NCT00441298) was reviewed and approved by the University of KwaZulu-Natal's Biomedical Research Ethics Committee (BREC ref. E 111/06), the South Africa Medicines Control
Council (MCC ref. 20060835), and Family Health International's Protection of Human Subjects Committee (PHSC ref. 9946). Before enrollment into the study, written informed consent was obtained from each participant.

Outcome measures of this analysis included contraceptive uptake and use patterns, pregnancy rates and pregnancy outcomes, and time off study product. Contraception and pregnancy data were entered onto standardized case report forms at the study sites and were faxed into the CAPRISA Data Management Centre using the DataFax system. Data on contraceptive method at each visit starting from screening, pregnancy diagnosis date, date of study product withdrawal and resumption, and pregnancy outcomes 
Table 2. Contraceptive Method Uptake at Baseline and Use at Study Exit by Site

\begin{tabular}{|c|c|c|c|c|c|c|}
\hline & \multicolumn{2}{|c|}{ Vulindlela $(n=611)$} & \multicolumn{2}{|c|}{ eThekwini $(n=278)$} & \multicolumn{2}{|c|}{ Total $(\mathrm{n}=\mathbf{8 8 9})$} \\
\hline & Baseline & Exit & Baseline & Exit & Baseline & Exit \\
\hline DMPA & $442(72.34)$ & 435 (71.19) & $140(50.36)$ & $119(42.81)$ & $582(65.47)$ & $554(62.32)$ \\
\hline NET-EN & $66(10.80)$ & $73(11.95)$ & $82(29.50)$ & $76(27.34)$ & $148(16.65)$ & $149(16.76)$ \\
\hline $\mathrm{COC}$ & $89(14.57)$ & $63(10.31)$ & $49(17.63)$ & 55 (19.78) & $138(15.52)$ & $118(13.27)$ \\
\hline Tubal ligation & $13(2.13)$ & $13(2.13)$ & $7(2.52)$ & $7(2.52)$ & $20(2.25)$ & $20(2.25)$ \\
\hline Hysterectomy & $1(0.16)$ & $1(0.22)$ & $0(0.0)$ & $0(0.0)$ & $1(0.11)$ & $1(0.11)$ \\
\hline No method & $0(0.0)$ & $26(4.26)$ & $0(0.0)$ & $21(7.55)$ & $0(0.0)$ & $47(5.29)$ \\
\hline All methods & $611(100.0)$ & $585(95.74)$ & $278(100.0)$ & $257(92.45)$ & $889(100.0)$ & $842(94.71)$ \\
\hline
\end{tabular}

DMPA, depot medroxyprogesterone acetate; NET-EN, norethisterone enanthate; COC, combined oral contraceptive.

Data are $\mathrm{n}(\%)$.

were captured and entered into the study database. Pregnancy outcomes documented included miscarriages, terminations of pregnancy, stillbirths, preterm live births with and without congenital anomalies, and full-term live births with and without congenital anomalies. Adverse maternal outcomes were also documented as adverse events.

For the first part of the analysis, contraceptive method groups were defined according to baseline method used and did not take into account method switching, discontinuation, missed visits, or contraceptives obtained from other external sources. We calculated incidence rates of pregnancy per 100 womanyears of observation for each contraceptive category considering method used up to the time of index pregnancy, thereby excluding pregnancy periods. This latter part of the analysis considered method switching, discontinuation, and missed study visits. Estimated date of pregnancy, in days, was calculated as the midpoint between the date of the first positive pregnancy test and the date of the previous negative pregnancy test. For women who had a pregnancy outcome with no pregnancy testing or women with several missed visits before a positive pregnancy test, the pregnancy start date was defined as 14 days after the last normal menstrual period. For women who did not become pregnant during the study, the censoring date was the last date with a negative pregnancy test on or before study termination date. Duration of time on study (in months) was calculated from randomization to estimated date of pregnancy or date of withdrawal or termination from study, whichever occurred first. In women with two pregnancy diagnoses during study follow-up period, only the first pregnancy was included in Kaplan-Meier analysis. Univariable and multivariable Cox proportional hazards analysis was used to examine baseline factors associated with incident pregnancy.

Statistical analysis was done using SAS 9.1.3. All statistical tests were two-sided and done at 5\% level of significance. Fisher's exact or chi-square tests were used for testing associations between categorical data. A Poisson distribution was assumed for $95 \%$ confidence intervals (CIs) of pregnancy rates ${ }^{14}$ and incidence rate ratios. Unpaired $t$ test and Wilcoxon rank sum twosample tests were performed where appropriate.

\section{RESULTS}

Of 2,160 women screened, 51 were screened out for pregnancy-related indications: 36 were already pregnant and 15 were planning to become pregnant sometime during the study duration. Twenty-six women were not on an eligible method of contraception. Nine hundred ninety-eight women were excluded for other nonpregnancy- or contraceptiverelated indications. After randomization, 196 women were excluded from the analysis for ineligibility based on pre-enrollment criteria. ${ }^{12} \mathrm{~A}$ total of 889 nonpreg-

Table 3. Pregnancy Rates by Study Arm and Study Site

\begin{tabular}{|c|c|c|c|c|c|}
\hline \multirow[b]{2}{*}{ Variable } & \multicolumn{2}{|c|}{ Vulindlela $(n=34)$} & \multicolumn{2}{|c|}{ eThekwini ( $n=19$ ) } & \multirow[b]{2}{*}{ Total $(n=53)$} \\
\hline & Tenofovir & Placebo & Tenofovir & Placebo & \\
\hline No. of pregnancies & 14 & 20 & 8 & 11 & 53 \\
\hline $\begin{array}{l}\text { Pregnancy incidence per } \\
100 \text { woman-years }\end{array}$ & 2.90 & 4.30 & 4.07 & 5.62 & 3.95 \\
\hline Incidence rate ratio & \multicolumn{2}{|c|}{0.67} & \multicolumn{2}{|c|}{0.72} & 0.69 \\
\hline Confidence interval $(P)$ & \multicolumn{2}{|c|}{$.31-1.40(.25)$} & \multicolumn{2}{|c|}{$.25-1.98(.49)$} & $.38-1.23(.18)$ \\
\hline
\end{tabular}




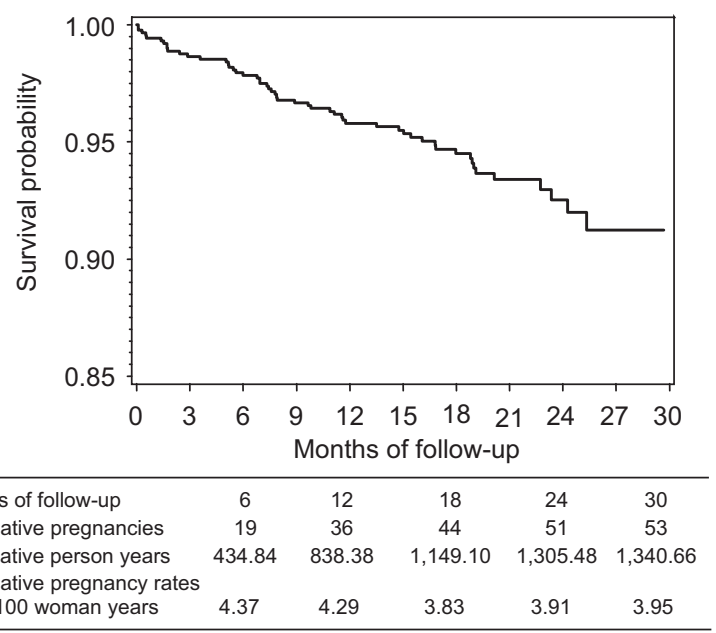

Fig. 2. Kaplan-Meier survival curve for pregnancy in the Centre for the AIDS Programme of Research in South Africa (CAPRISA) 004 trial.

Sibeko. Contraceptive Choices and Pregnancy Rates. Obstet Gynecol 2011.

nant women were eligibly enrolled in the CAPRISA 004 trial and were included in this analysis (Fig. 1).

Women from the urban site were older, more likely to have a stable partner, and had a lower monthly income. Women at the rural site reported fewer lifetime sexual partners (2.1 compared with $6.0 ; P<.001)$ and had younger partner(s) in the 30 days before enrollment (26.4 compared with $39.6 ; P<.001) .{ }^{13}$ The mean age of the participants was 23.9 years. Three fourths of the women were multiparous, almost all were in stable relationships, few reported having a casual partner in the 30 days before enrollment, and $71 \%$ reported inconsistent condom use (Table 1).

All women were using a nonbarrier method of contraception at the time of enrollment into the study; $730(82.12 \%)$ chose an injectable form of contraceptive, $138(15.52 \%)$ chose combined oral contraceptives, and 21 (2.36) had undergone female sterilization (20 tubal ligations and one hysterectomy) (Table 2). Depot-medroxyprogesterone acetate was a most popular injectable method, used by more women than norethisterone enanthate throughout the follow-up period. Overall, method switching was uncommon and contraceptive use remained high throughout the study. At study exit, 47 (5.29\%) of participants reported not using any contraceptive method.

At the end of 30 months of study follow-up, the overall pregnancy incidence rate was 3.95 per 100 woman-years (95\% CI 2.96-5.17), somewhat higher in the urban than the rural cohorts (incidence rate ratio $0.75,95 \%$ CI $0.41-1.37$, z-test $P=.30$ ). Although not significantly different, pregnancy rates were

Table 4. Participant Profile, Number of Pregnancies, and Pregnancy Rates by Contraceptive Method at Baseline

\begin{tabular}{|c|c|c|c|c|c|}
\hline Variable & $\begin{array}{c}\text { DMPA } \\
(\mathrm{n}=582)\end{array}$ & $\begin{array}{l}\text { NET-EN } \\
(\mathrm{n}=148)\end{array}$ & $\begin{array}{c}\text { COC } \\
(n=138)\end{array}$ & $\begin{array}{c}\text { Other } \\
(n=21)\end{array}$ & Total \\
\hline \multicolumn{6}{|l|}{ Age group (y) } \\
\hline $18-24$ & $398(68.38)$ & $105(70.95)$ & $74(53.62)$ & $2(9.52)$ & 579 \\
\hline $25-29$ & $112(19.24)$ & $29(19.59)$ & $32(23.19)$ & $4(19.05)$ & 177 \\
\hline $30-34$ & $44(7.56)$ & $10(6.76)$ & $22(15.94)$ & $9(42.86)$ & 85 \\
\hline 35 or older & $28(4.81)$ & $4(2.70)$ & $10(7.25)$ & $6(28.57)$ & 48 \\
\hline Median (IQR) & $22(20-26)$ & $22(20-25)$ & $24(20-29)$ & $33(29-35)$ & \\
\hline \multicolumn{6}{|l|}{ Parity } \\
\hline 0 & $91(15.64)$ & $51(34.46)$ & $55(39.86)$ & $0(0.0)$ & 197 \\
\hline 1 & 349 (59.97) & $71(47.97)$ & $50(36.23)$ & $0(0.0)$ & 470 \\
\hline 2 & $100(17.18)$ & $20(13.51)$ & $23(16.67)$ & $4(19.05)$ & 147 \\
\hline 3 & $29(4.98)$ & $3(2.03)$ & $8(5.80)$ & $10(47.62)$ & 50 \\
\hline 4 or more & $13(2.23)$ & $3(2.03)$ & $2(1.45)$ & $7(33.33)$ & 25 \\
\hline \multicolumn{6}{|l|}{ Pregnant } \\
\hline Negative & 570 (97.94) & 145 (97.97) & $100(72.46)$ & $21(100.0)$ & 836 \\
\hline Positive & $12(2.06)$ & $3(2.03)$ & $38(27.54)$ & $0(0.0)$ & 53 \\
\hline $\begin{array}{l}\text { Pregnancy incidence rate per } \\
100 \text { woman-years }(95 \% \mathrm{Cl})\end{array}$ & $1.34(0.69-2.35)$ & $1.37(0.28-3.99)$ & $19.73(13.96-27.08)$ & & \\
\hline \multicolumn{6}{|l|}{ Pregnancy rate* } \\
\hline $\begin{array}{l}\text { Pregnancy incidence rate per } \\
100 \text { woman-years }(95 \% \mathrm{Cl})\end{array}$ & $0.46(0.13-1.18)$ & $1.75(0.48-4.49)$ & $11.47(7.27-17.22)$ & & \\
\hline
\end{tabular}




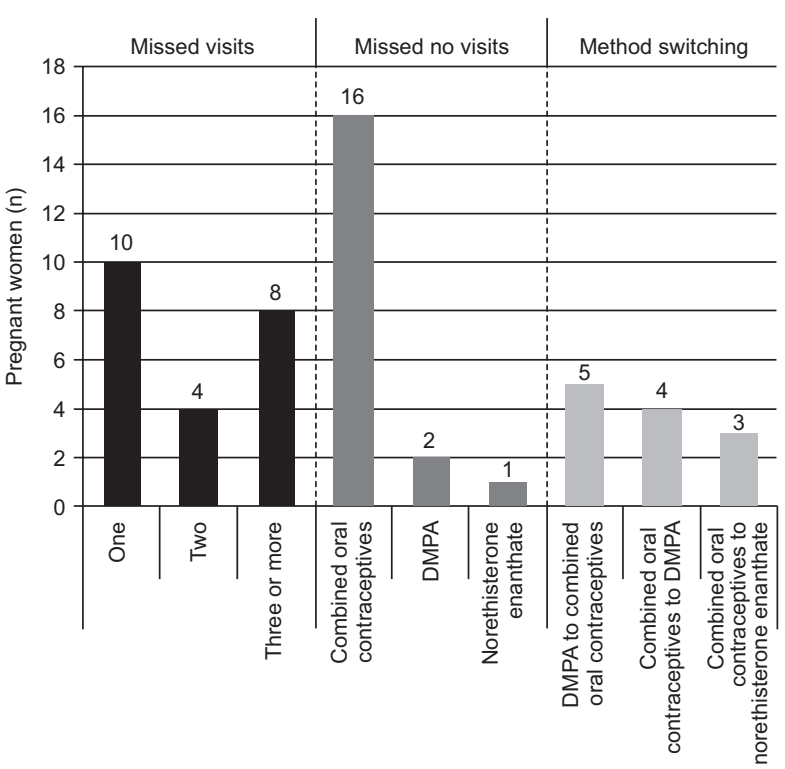

Fig. 3. Clinic attendance and contraceptive use patterns among women who became pregnant $(n=53)$ in the Centre for the AIDS Programme of Research in South Africa (CAPRISA) 004 trial. DMPA, depot medroxyprogesterone acetate.

Sibeko. Contraceptive Choices and Pregnancy Rates. Obstet Gynecol 2011.

slightly lower in the tenofovir gel arm than the placebo arm (z-test $P=.18$; Table 3). Pregnancy rates remained somewhat static at approximately four per 100 woman-years from beginning through to study completion (Fig. 2).

By study end, 54 pregnancies were observed among 53 women who had 55 outcomes between them (Fig. 1). One participant was pregnant twice and another had a twin pregnancy. Participants who became pregnant had similar baseline characteristics as those who did not become pregnant except for level of education: $97.2 \%$ had a high school education compared with $88.2 \%$ in the nonpregnant group.

Among the 54 pregnancies, $10(18.52 \%)$ resulted in miscarriages (including four probable chemical and spurious pregnancies), six $(11.11 \%)$ were terminated (including one noninvasive mole), $35(64.81 \%)$ resulted in full-term live deliveries (two early neonatal deaths), and four $(7.41 \%)$ resulted in preterm deliveries (one early neonatal death) (Fig. 1). All three children with diagnosed fetal distress syndrome were in the placebo arm. There were no stillbirths. All early neonatal deaths were in the placebo arm. There were no congenital abnormalities observed.

Of the 39 viable pregnancies, three delivered by cesarean delivery (two in the tenofovir arm and one in the placebo arm), one had postpartum hemorrhage, and one had premature rupture of membranes. The latter two were allocated to the tenofovir gel arm.

As expected, pregnancy rates based on category of contraceptive method used at baseline were 19 times higher among combined oral contraceptive users than among injectable contraceptive users with no distinct differences between depot-medroxyprogesterone acetate and norethisterone enanthate users (Table 4). However, taking method switching and discontinuation into consideration, the pregnancy rates were lowest among depot-medroxyprogesterone acetate users $(0.46$ per 100 woman years, 95\% CI $0.13-1.18)$ followed by norethisterone enanthate $(1.75$ per 100 woman years, 95\% CI $0.48-4.49)$ and combined oral contraceptive (11.47 per 100 woman years, 95\% CI 7.27-17.22) users.

Pregnancies occurred in women who either missed study visits, thus discontinuing method use (22 of 53 [41.51\%]), or did not miss study visits but were on combined oral contraceptives (19 of 53 [35.85\%]), or switched contraceptive methods (12 of 53 [22.64\%]) (Fig. 3).

Three women who became pregnant also became HIV-infected during the study, all in the placebo arm. Two of these pregnancies were diagnosed before HIV seroconversion.

Method of contraception was the only baseline factor that was significantly associated with pregnancy in both the univariable and multivariable proportional hazard models. The adjusted hazard ratio for combined oral contraceptive use was 15.91 (95\% CI 8.03-31.52, $P<.001$; Table 5). Inconsistent condom use in the past 30 days was also a significant predictor of pregnancy with a hazard ratio of 2.05 (1.04-4.04) in the multivariable model.

Total person-time of observation was 1,340.7 woman-years. Time off product as a result of pregnancies was 20.9 woman-years $(1.56 \%$ of total persontime), mostly attributable to the full-term births. The median time between date of last product use and pregnancy detection was 14 days (range 0-92 days).

\section{DISCUSSION}

The high contraceptive use throughout follow-up in this trial resulted in a low overall pregnancy rate of 3.95 per 100 woman-years. This pregnancy rate was lower compared with 17.7 per 100 woman-years that was observed in the CAPRISA 050/051 microbicide preparedness study, a study conducted without the implementation of the contraceptive curriculum. ${ }^{15}$ The observed pregnancy rate in the CAPRISA 004 trial was also lower than previous microbicide studies, 
Table 5. Baseline Predictors of Pregnancy in Cox Regression Models

\begin{tabular}{|c|c|c|c|c|}
\hline \multirow[b]{2}{*}{ Variable } & \multicolumn{2}{|c|}{ Univariable } & \multicolumn{2}{|c|}{ Multivariable } \\
\hline & $\mathrm{HR}(95 \% \mathrm{CI})$ & $P$ & HR $(95 \% \mathrm{CI})$ & $P$ \\
\hline \multicolumn{5}{|l|}{ Site } \\
\hline Vulindlela & Referent & & Referent & \\
\hline eThekwini & $1.33(0.76-2.34)$ & .32 & $1.9(0.97-4.03)$ & .06 \\
\hline \multicolumn{5}{|l|}{ Age group (y) } \\
\hline $18-24$ & Referent & & Referent & \\
\hline $25-29$ & $1.19(0.62-2.31)$ & .60 & $0.86(0.43-1.72)$ & .66 \\
\hline $30-34$ & $1.61(0.74-3.48)$ & .23 & $0.85(0.34-2.12)$ & .73 \\
\hline 35 or older & N/A & & & \\
\hline \multicolumn{5}{|l|}{ Type of contraception } \\
\hline DMPA & Referent & & Referent & \\
\hline NET-EN & $1.01(0.29-3.59)$ & .98 & $0.86(0.24-3.11)$ & .81 \\
\hline Oral & $14.51(7.58-27.78)$ & $<.0001$ & $15.91(8.03-31.52)$ & $<.0001$ \\
\hline \multicolumn{5}{|l|}{ Marital status } \\
\hline Other & Referent & & Referent & \\
\hline Married & $1.1(0.07-17.52)$ & .95 & $1.54(0.09-25.97)$ & .77 \\
\hline Stable & $3.57(0.49-25.87)$ & .20 & $3.59(0.47-27.62)$ & .22 \\
\hline \multicolumn{5}{|l|}{ Education } \\
\hline Primary school & Referent & & Referent & \\
\hline High school & $2.45(0.34-17.74)$ & .38 & $0.78(0.11-5.83)$ & .81 \\
\hline Tertiary & $5.31(0.65-43.18)$ & .12 & $0.76(0.08-6.92)$ & .81 \\
\hline \multicolumn{5}{|l|}{ Number of previous live births } \\
\hline Previous live births (per one live birth increase) & $0.71(0.51-0.995)$ & $<.047$ & $0.95(0.65-1.40)$ & .81 \\
\hline \multicolumn{5}{|l|}{ Sex acts in past $30 \mathrm{~d}$} \\
\hline Sex acts in last $30 \mathrm{~d}$ (per one act increase) & $1.0(0.98-1.03)$ & .73 & $1.01(0.98-1.05)$ & .53 \\
\hline \multicolumn{5}{|l|}{ Condom use in past $30 \mathrm{~d}$} \\
\hline Always & Referent & & Referent & \\
\hline Inconsistent & $1.29(0.69-2.42)$ & .42 & $2.05(1.04-4.04)$ & .04 \\
\hline
\end{tabular}

$\mathrm{HR}$, hazard ratio; $\mathrm{Cl}$, confidence interval; N/A, not applicable; DMPA, depot medroxyprogesterone acetate; NET-EN, norethisterone enanthate; COC, combined oral contraceptive.

even after procedures were revised to dispense contraceptives at the study sites. ${ }^{16}$ The low pregnancy rate observed in the CAPRISA 004 trial suggests that the contraceptive curriculum was effective in reducing pregnancy rates. Onsite method provision and study visits synchronized with injectable administration further enhanced compliance with the method. Most importantly, CAPRISA 004 was the first trial to include an eligibility criterion requiring effective contraceptive initiation and provide effective methods onsite. Future biomedical prevention trials should consider including a comprehensive contraception strategy to effectively reduce pregnancy rates.

Although not statistically significant, pregnancy incidence rates were higher in the tenofovir gel arm. Although tenofovir gel is not known to reduce probability of conception, there needs to be vigilance in evaluating this association in future tenofovir trials.

Pregnancies that did occur in the CAPRISA 004 trial were mainly among combined oral contraceptive users. The pregnancy rate was more than 10-fold higher in combined oral contraceptive users than depot-medroxyprogesterone acetate users, and combined oral contraceptive use was the most important predictor of pregnancy in this trial, a finding consistent with findings in the HPTN 039 study. ${ }^{17}$ Inadvertent pregnancies in pill users are not uncommon and often involve pill-taking errors, although many factors may contribute to "pill failure." 18 Data from the United States indicate that pregnancy rates within the first year of perfect method use are the same for combined oral contraceptives and depot-medroxyprogesterone acetate at $0.3 \%$. However, by 12 months, the pregnancy rate with typical use for combined oral contraceptives is $8 \%$ compared with $3 \%$ for depot-medroxyprogesterone acetate. ${ }^{19}$ The combined oral contraceptive pregnancy rate was higher still in this trial.

Pregnancies occurred with higher frequency among women who missed one or more study visits or switched methods. For future microbicide studies, researchers need to prioritize these women and develop a strategy of timely identification and providing targeted care and counseling in dealing with women 
who miss method replenishment dates and carefully manage timing of switching to minimize pregnancies.

Exposure to tenofovir gel during pregnancy was limited (median 14 days) and was not associated with adverse outcomes in this trial. Most pregnancies resulted in a full-term live birth. The three neonatal deaths in this study were all in the placebo arm. Additionally, no congenital anomalies were noted in the 39 newborns that were delivered alive (both preterm and full-term) and assessed in the early postpartum period. Potential teratogenicity was minimized by optimizing frequency of pregnancy testing using a highly sensitive testing assay and thus reducing the period of potential drug exposure. These findings, albeit after very short exposure to the drug and small sample size, are in keeping with those of the Tenofovir Antiretroviral Pregnancy Registry, which shows no evidence of higher rates of congenital anomalies after systemic exposure in patients on treatment. ${ }^{11}$ Furthermore, these findings are similar to nonhuman studies in which there were no demonstrable teratogenic effects up to 5 years after birth, postexposure to chronic low-dose tenofovir in pregnancy.$^{20}$ As a result of high HIV seroconversion rates late in pregnancy, the safety of continuous gel use throughout pregnancy needs to be evaluated further.

Product withdrawal resulting from pregnancy accounted for only $1.6 \%$ of total person-time in CAPRISA 004 with negligible statistical effect. It has been shown that frequent pregnancy testing and using highly sensitive pregnancy tests to diagnose pregnancy in HIV prevention trials may lead to many false-positive or chemical pregnancies. Consequently, women may be withdrawn from product for unnecessary prolonged periods of time, thus compromising the power of the study. ${ }^{21}$ To overcome this limitation, CAPRISA 004 successfully implemented a strategy to minimize false-positive or chemical pregnancies. Additionally, women continued study follow-up after a pregnancy diagnosis until the pregnancy outcome was established, allowing for timely product resumption on pregnancy end.

The short duration of exposure to drug in utero made it difficult to assess the effect of tenofovir gel on pregnancy outcomes. In addition, high contraceptive use rates and low pregnancy rates in CAPRISA 004 may have been the result of self-selection of women not intending to fall pregnant into the study rather than the effect of the contraceptive curriculum per se, thus limiting generalizability of the results.

Strengths of this analysis include good contraceptive data; accurate and frequent testing for the pregnancy outcome; excellent retention; and a large enough study sample size to yield an adequate number of pregnancy outcomes.

The comprehensive contraceptive curriculum, including prespecified contraceptive and pregnancyrelated eligibility criteria developed for the CAPRISA 004 tenofovir gel trial, was an effective strategy for enhancing contraceptive use and reducing pregnancy rates in a microbicide trial. The pregnancy rate was low and no safety concerns arose with the use of tenofovir gel. As is the case in general use, injectable methods were more effective in preventing pregnancy than combined oral contraceptives. If the safety and effectiveness of tenofovir gel are confirmed, contraceptive use must be a prominent component of future prevention trials. Additionally, safety studies on prolonged tenofovir gel use throughout pregnancy should be prioritized because HIV prevention in pregnancy is important to reduce maternal morbidity and mortality, mother-to-child transmission, and infant mortality.

\section{REFERENCES}

1. UNAIDS. UNAIDS report on the global AIDS epidemic 2010. Available at: www.unaids.org/globalreport/. Geneva: Joint United Nations Programme on HIV/AIDS; 2010. Retrieved February 11, 2011.

2. UNAIDS. 2008 report on the global AIDS epidemic update. Availableat:www.unaids.org/en/KnowledgeCentre/HIVData/ GlobalReport/2008/2008_Global_report.asp. Geneva: Joint United Nations Programme on HIV/AIDS; 2008. Retrieved October 31, 2008.

3. Padian NS, McCoy SI, Balkus JE, Wasserheit JN. Weighing the gold in the gold standard: challenges in HIV prevention research. AIDS 2010;24:621-35.

4. AVAC. Ongoing and planned PreExposure Prophylaxis (PrEP) trials (February 2011). Available at: www.avac.org/ht/ a/GetDocumentAction/i/3113. Retrieved February 2011.

5. Raymond EG, Taylor D, Cates W Jr, Tolley EE, Borasky D, Cancel A, et al. Pregnancy in effectiveness trials of HIV prevention agents. Sex Transm Dis 2007;34:1035-9.

6. Lagakos SW, Gable AR, eds. Methodological challenges in biomedical HIV prevention trials. Washington, DC: National Academy of Sciences; 2008.

7. Smart T. Microbicides 2006: high rates of pregnancy pose challenges for microbicide trials. Available at: www.aidsmap. com/en/news/3F76DCE3-83FA-4523-A920-5CB0829DD33A. asp. Retrieved June 2006.

8. Van Damme L, Govinden R, Mirembe FM, Guedou F, Solomon S, Becker ML, et al. Lack of effectiveness of cellulose sulfate gel for the prevention of vaginal HIV transmission. N Engl J Med 2008;359:463-72.

9. Peterson L, Nanda K, Opoku BK, Ampofo WK, OwusuAmoako M, Boakye AY, et al. SAVVY (C31G) gel for prevention of HIV infection in women: a Phase 3, doubleblind, randomized, placebo-controlled trial in Ghana. PLoS One 2007;2:e1312.

10. Feldblum PJ, Adeiga A, Bakare R, Wevill S, Lendvay A, Obadaki F, et al. SAVVY vaginal gel (C31G) for prevention of HIV infection: a randomized controlled trial in Nigeria. PLoS One 2008;3:e1474. 
11. Puoti M, Brown RS Jr, Goodwin D, Zhang S, Fagan E. Tenofovir disoproxil fumarate in pregnancy: findings from the antiretroviral pregnancy registry. Dig Liver Dis 2009;41:A39-40.

12. Abdool Karim Q, Abdool Karim SS, Frohlich JA, Grobler AC, Baxter C, Mansoor LE, et al. Effectiveness and safety of tenofovir gel, an antiretroviral microbicide, for the prevention of HIV infection in women. Science 2010;329:1168-74.

13. Karim QA, Kharsany AB, Frohlich JA, Baxter C, Yende N, Mansoor LE, et al. Recruitment of high risk women for HIV prevention trials: baseline HIV prevalence and sexual behavior in the CAPRISA 004 tenofovir gel trial. Trials 2011;12:67.

14. McCullagh P, Nelder JA. Generalized linear models. London (UK): Chapman and Hall, Inc; 1983.

15. Abdool Karim Q, Kharsany AB, Frohlich JA, Werner L, Mashego M, Mlotshwa M, et al. Stabilizing HIV prevalence masks high HIV incidence rates amongst rural and urban women in KwaZulu-Natal, South Africa. Int J Epidemiol 2010 Nov 3 [epub ahead of print].

16. Skoler-Karpoff S, Ramjee G, Ahmed K, Altini L, Plagianos MG, Friedland B, et al. Efficacy of Carraguard for prevention of HIV infection in women in South Africa: a randomised, double-blind, placebo-controlled trial. Lancet 2008;372: 1977-87.

17. Reid SE, Dai JY, Wang J, Sichalwe BN, Akpomiemie G, Cowan FM, et al. Pregnancy, contraceptive use, and HIV acquisition in HPTN 039: relevance for HIV prevention trials among African women. J Acquir Immune Defic Syndr 2010;53:606-13.

18. Fraser IS, Jansen RP. Why do inadvertent pregnancies occur in oral contraceptive users? Effectiveness of oral contraceptive regimens and interfering factors. Contraception 1983;27: $531-51$.

19. Trussell J. Contraceptive failure in the United States. Contraception 2004;70:89-96.

20. Van Rompay KKA, Durand-Gasselin L, Brignolo LL, Ray AS, Abel K, Cihlar T, et al. Chronic administration of tenofovir to rhesus macaques from infancy throughout adulthood and pregnancy: summary of pharmacokinetics, biological and virological effects. Antimicrob Agents Chemother 2008;52:3144-60.

21. Schreiber CA, Sammel M, Hillier SL, Barnhart KT. A little bit pregnant: modeling how the accurate detection of pregnancy can improve HIV prevention trials. Am J Epidemiol 2009;169: 515-21.

\section{Continuing Medical Education Credits Available for the Clinical Expert Series}

Continuing medical education (CME) credits are available for reading the Clinical Expert Series. Visit http://www.greenjournal.org and click on the "CME" tab to read the articles and complete their associated quizzes.

\section{ACCME Accreditation}

The American College of Obstetricians and Gynecologists is accredited by the Accreditation Council for Continuing Medical Education (ACCME) to provide continuing medical education for physicians.

\section{AMA PRA Category 1 Credit(s) ${ }^{\mathrm{TM}}$}

The American College of Obstetricians and Gynecologists designates this journal-based CME activity for a maximum of 2 AMA PRA Category 1 Credits. ${ }^{\mathrm{TM}}$ Physicians should claim only the credit commensurate with the extent of their participation in the activity.

\section{College Cognate Credit(s)}

The American College of Obstetricians and Gynecologists designates this journal-based CME activity for a maximum of 2 Category 1 College Cognate Credits. The College has a reciprocity agreement with the AMA that allows AMA PRA Category 1 Credits $^{\mathrm{TM}}$ to be equivalent to College Cognate Credits. 